\title{
Éduquer scolairement dans un monde pluraliste : opportunité d'une approche normative du cadre réglementaire
}

Educate Academically in a Pluralistic World: Opportunity of a Normative Approach of the Regulatory Framework

\section{Vincent Lorius}

\section{OpenEdition}

\section{Journals}

Édition électronique

URL : http://journals.openedition.org/pratiques/2249

DOI : $10.4000 /$ pratiques.2249

ISSN : 2425-2042

\section{Éditeur}

Centre de recherche sur les médiations (CREM)

Édition imprimée

Date de publication : 31 décembre 2014

Référence électronique

Vincent Lorius, «Éduquer scolairement dans un monde pluraliste : opportunité d'une approche normative du cadre réglementaire », Pratiques [En ligne], 163-164 | 2014, mis en ligne le 31 décembre 2014, consulté le 03 mai 2019. URL : http://journals.openedition.org/pratiques/2249; DOI : 10.4000/ pratiques.2249

Ce document a été généré automatiquement le 3 mai 2019.

(C) Tous droits réservés 


\section{Éduquer scolairement dans un monde pluraliste : opportunité d'une approche normative du cadre réglementaire}

Educate Academically in a Pluralistic World: Opportunity of a Normative Approach of the Regulatory Framework

\section{Vincent Lorius}

\section{Introduction}

1 Pour D. Weinstock (2006), c'est le double constat de l'importance des décisions morales privées qui sont prises dans des cadres institutionnels, ainsi que le caractère souvent indécidable de certaines controverses morales au sein d'une société démocratique, qui doit nous faire considérer comme importantes les questions morales institutionnelles. Ces observations s'appliquent assez directement au domaine scolaire dans la mesure où l'école affecte les questions privées (la vie des familles, leur emploi du temps, les relations entre parents et enfants...) et est dépositaire de l'immense pouvoir de faciliter ou non l'insertion des plus jeunes par son quasi-monopole des opérations de certification. Par ailleurs, elle doit proposer des modalités permettant le vivre et l'apprendre ensemble malgré la diversité des opinions, en particulier des opinions morales.

2 L'objectif de cet article est de proposer quelques éléments en faveur de la thèse selon laquelle, dans ce contexte, le pluralisme moral, non seulement n'est pas un obstacle à l'activité des éducateurs scolaires, mais constitue probablement l'une des conditions de son déploiement. Cette thèse, pour être établie, nécessite plusieurs étapes. La première est de donner une définition de l'éducation scolaire permettant de justifier le recours au minimalisme moral comme possibilité pour penser l'activité éthique des éducateurs scolaires. Nous pourrons alors qualifier ce que nous entendons par pluralisme moral (c'est-à-dire incluant des conceptions minimalistes et maximalistes de l'éthique) et en 
quoi celui-ci peut être une composante possible, voire nécessaire, de l'éducation scolaire. Cette deuxième étape prendra appui sur des entretiens réalisés avec des professionnels de l'éducation et visera à faire apparaitre empiriquement des traces, dans leurs discours, d'un pluralisme d'exploration comme moyen de faire face aux situations professionnelles. Ceci permettra alors, en guise d'illustration, d'envisager comment une lecture pluraliste des textes réglementaires peut contribuer à un meilleur positionnement.

\section{Éducation scolaire et pluralisme : une rencontre impossible?}

\section{L'éducation scolaire comme activité d'imputation}

3 L'éducation scolaire se définit par un cadre normatif pluriel. Elle est bornée par des règles qui portent pour certaines d'entre elles sur les finalités à poursuivre ou les procédures à respecter. Elle est également impactée par les normes ${ }^{1}$ privées des acteurs qui, qu'ils soient professionnels ou usagers, ont bien évidement toutes sortes de croyances et convictions relatives à ce que doit être la scolarité. Face à ce constat, deux attitudes au moins sont possibles : soit considérer que les normes scolaires légitimes sont celles définies par l'une de ces sources (par exemple le ministère de l'Éducation nationale, ou les familles ${ }^{2}$ ), soit prendre acte de cette pluralité normative pour la considérer constitutive du procès d'éducation scolaire. Dans cet article, nous soutiendrons l'opportunité de la seconde hypothèse : il serait peut-être possible de justifier ce choix pour des raisons de droit (en posant par exemple la supériorité normative du pluralisme), mais nous nous contenterons de raisons de fait. La simple observation du fonctionnement quotidien de l'école montre en effet que domine un cadre normatif pluriel, à la fois une cause et conséquence de l'impossibilité de fixer des objectifs ultimes à l'éducation (Kerlan, 2003).

Pour comprendre comment les éducateurs scolaires font face à cet état de fait, nous pouvons commencer par observer l'énergie et le temps qu'ils passent à intervenir auprès de «tiers" dans le but faciliter les apprentissages de leurs élèves. Ces tiers sont bien évidement et en premier lieu les parents, mais peuvent aussi être des partenaires éducatifs (associations, structures de soutien scolaire) ou même des membres de l'établissement scolaire (assistants d'éducation...). Ainsi, on ne compte plus les dispositifs d'implication des parents dans le processus d'enseignement (voir, par exemple, l'école des parents, dispositif récent de "mallette des parents " promue par le ministère de l'Éducation nationale ${ }^{3}$ ). Par ailleurs, à l'intérieur même des établissements, de nombreux rapports relèvent, pour le regretter, des phénomènes de sous-traitance ${ }^{4}$ de la difficulté scolaire aux dispositifs de soutien organisés dans le temps scolaire même, et pour lesquels les enseignants sont amenés à définir des modalités de répartition des élèves, de définition des objectifs, et à engager des opérations de mise en cohérence entre ces dispositifs et l'enseignement dispensé en classe. Il semble donc bien exister un fort différentiel entre l'ensemble des tâches relevant d'une action directe de l'enseignant sur les élèves et l'ensemble de celles qui caractérisent l'activité des éducateurs (s'investir dans un travail d'équipe et dans un projet d'établissement, coordonner les activités des intervenants extérieurs, s'impliquer dans un dialogue avec les parents, les élus, les associations, etc.). 
5 Si l'activité des professionnels de l'école ne peut se concevoir sous le seul angle d'une action de l'éducateur sur l'éduqué, mais intègre aussi les actions effectuées dans ce but à travers des tiers, il nous faut proposer une définition de l'éducation scolaire permettant cet élargissement. Pour cela, nous pouvons considérer que les décisions professionnelles reposent sur une évaluation du rôle que peuvent jouer les personnes impliquées dans la prise en charge scolaire (élèves, éducateur, parents, partenaires). On peut en effet constater que, par exemple face aux difficultés rencontrées par certains élèves, les solutions envisagées peuvent concerner l'élève lui-même (remobilisation, exhortation, dispositifs d'aides diverses...), l'équipe éducative (modification des formes de prises en charge, modification des contenus à transmettre, des objectifs), les parents (par exemple en visant la « responsabilisation» des parents, ou en donnant des consignes aux parents pour la réalisation des devoirs ou même pour l'organisation périscolaire et en particulier des temps de repos), des intervenants extérieurs (soutien à la parentalité, soutien scolaire, soutien éducatif).

6 On peut donc penser que les professionnels de l'éducation scolaire s'attachent à savoir ce qui peut relever, plus particulièrement, à chaque instant, de la responsabilité de l'élève et de son environnement humain, pour en faire l'objet de leur action. Cette dimension du travail éducatif est particulièrement prégnante dans les relations des éducateurs avec les parents. On ne peut en effet que constater, même si les échanges sont parfois difficiles, que les parents sont pris en compte par les éducateurs dans leur pratique quotidienne pour des raisons évidentes d'efficacité mais également pour des raisons réglementaires qui font que, par exemple, les bénéficiaires de l'école ont légitimement droit à des recours contre des pratiques professionnelles qu'ils jugeraient inadaptées ou non conformes.

7 Une définition de l'éducation scolaire comme "activité d'imputation des leviers de l'apprentissage » pour décrire les prises en charge scolaire dans l'école française d'aujourd'hui présente donc selon nous plusieurs avantages. En premier lieu, cette proposition permet de positionner dans le champ de l'éducation scolaire des tâches extrêmement variées (d'enseignement, d'accompagnement, de remobilisation, de contrôle...) qui autorisent l'intégration dans un même ensemble des éducateurs scolaires quelles que soient leurs fonctions à partir du moment où ils sont au contact quotidien des usagers de l'école. En second lieu, elle permet de prendre en compte la possibilité pour les éducateurs d'imputer ce qui permettra l'apprentissage, de façon variée suivant les situations, à l'élève en premier lieu, mais également aux autres acteurs susceptibles de faciliter ce projet. Ces imputations sont bien évidemment non exclusives et, dans la plupart des cas, plusieurs personnes seront concernées en plus de l'élève pour l'aider, faciliter son travail, lui donner du sens. Il est difficile de nier que cette multi-attribution soit aujourd'hui une part importante du travail des éducateurs et cela implique que ces derniers sont tenus, de fait, à la prise en compte des conceptions, non seulement scolaires mais également morales, des usagers. Il découle de cela l'utilité de disposer d'éléments pour comprendre comment cette prise en compte peut se réaliser et il parait pour cela indispensable de se référer à une catégorisation des théories morales susceptibles d'être utilisées par les acteurs. 


\section{intérêt d'une conception minimale de l'éthique pour penser l'imputation scolaire}

R. Ogien (2007: 12) propose de classer en deux catégories les théories morales: «Le monde d'Aristote nous recommande tout un art de vivre et pas seulement un code de bonne conduite en société, comme celui de Kant, pour qui nous avons des devoirs moraux à l'égard d'autrui, mais aussi de nous-mêmes. J'appelle "maximaliste" un tel monde moral et, par contraste, "minimalistes" des mondes moraux moins envahissants». Plus précisément, l'auteur propose de considérer comme minimales les morales convenant de l'opportunité d'un principe de non-nuisance limitant les interventions aux cas de torts flagrants causés à autrui (Ogien, 2004 : 30).

Les théories morales peuvent donc être maximalistes ou minimalistes, et se différencient par la manière de qualifier moralement le vivre ensemble (comme le tout de l'éthique pour le minimaliste et comme l'une de ses composantes pour le maximaliste) et l'idée de non-nuisance (comme repère nécessaire et suffisant pour le minimaliste, comme repères parmi d'autres pour le maximaliste). Cette distinction revient à attribuer une valeur morale différente au rapport à soi-même : celui-ci peut être un objet du jugement moral (pour le maximaliste), ou non (pour le minimaliste). Pour ce dernier en effet, le rapport à soi-même est hors du champ du vivre ensemble et, par conséquent, le principe de nonnuisance ne le concerne pas.

Ce point d'appui théorique nous permet de formuler l'hypothèse selon laquelle la distinction minimalisme/maximalisme est à la fois possible et utile pour penser les questions d'éducation scolaire ${ }^{5}$. Pour la défendre, nous commencerons par examiner deux types d'objections pouvant être faites à cette proposition: celles de l'inadaptation du minimaliste au contexte scolaire ou à la possibilité même de l'éducation. Nous pourrions en effet considérer en première analyse que, le point de vue minimaliste étant totalement absent du cadre réglementaire organisant l'action des professionnels, il serait susceptible de mettre ces derniers en contradiction avec les missions qui leur sont confiées par l'institution. Les textes qui régissent l'action scolaire sont de fait largement portés sur la modification du rapport des élèves avec eux-mêmes ${ }^{6}$. Ils ne font jamais non plus mention de limites qu'il faudrait apporter aux dispositions prises en considérant par exemple que dans certains contextes, certains dispositifs pourraient «faire plus de mal que de bien ", et qui seraient l'expression d'un souci de non-nuisance. Cette première objection parait assez simple à lever. En effet, la demande institutionnelle relative aux questions éducatives doit être comprise pour ce qu'elle est : une déclaration d'intention qui dit aussi peu sur les objectifs que sur les moyens de les atteindre et pour lesquels l'institution se garde d'ailleurs bien de vérifier les mises en œuvre. Ceci ne réduit pas l'importance de ces prescriptions, mais indique simplement que leur prise en compte relève d'un processus classique au sein de l'Éducation nationale: il s'agit d'éléments de discours, constitutifs d'une culture institutionnelle, et dont l'opérationnalisation relève de l'éthique professionnelle des éducateurs, en comprenant éthique comme un arbitrage personnel sur ce qui constitue le bien. Cet arbitrage éthique induit souvent la prudence et le discernement dans la mise en œuvre des recommandations portant sur les attitudes. À titre d'exemple, que penser d'un éducateur qui, voulant respecter les prescriptions d'une éducation à la santé, reprocherait à un élève de ne pas être «bien reposé » le matin alors que les habitudes ou nécessité familiales imposent à tous les membres de sa famille un 
coucher tardif? Une approche minimaliste de l'éducation, qui fait prévaloir une attitude prudente face à la possibilité d'une intervention relative aux obligations envers euxmêmes que les élèves devraient respecter, est donc possible.

11 La deuxième objection que l'on pourrait faire à une approche minimaliste de l'éducation consisterait à poser que celle-ci induirait des comportements inadaptés en abandonnant, au motif de la non-nuisance, l'individu dont on a la charge, à lui-même. En d'autres termes, nous aurions le droit (voire le devoir) d'aider l'autre, quand bien même l'autre ne veut pas être aidé. Cette objection est sérieuse et la question des limites du consentement fait bien partie des critiques qui ont pu être formulées au minimalisme (Marzano, 2009). Si elle incite à la vigilance, cette objection ne détruit pas à elle seule la possibilité d'associer minimalisme et éducation et ce pour au moins deux raisons. D'abord parce ce que nous concevons l'éducation scolaire comme nécessitant de la part des éducateurs et à travers des processus d'imputation, des décisions concernant les élèves, mais également des décisions concernant leurs parents, dont le consentement doit être considéré différemment de celui d'un enfant. Ensuite, parce qu'il parait aussi stupide de toujours tenir compte de la volonté d'un enfant comme de ne jamais le prendre en compte: des positionnements minimalistes sont donc, sans doute, parfois envisageables.

12 Pour conclure provisoirement, on peut donc défendre l'idée qu'il existe des arguments indiquant que le minimalisme ne parait pas en lui-même et à priori inadapté à l'éducation scolaire, car contradictoire ni avec le cadre réglementaire, ni avec l'attention à porter aux bénéficiaires du service public d'éducation. De ce point de vue, l'éthique minimale ne rejette pas le recours aux principes moraux fondant la morale scolaire classique qui est d'inspiration maximaliste, elle en discute simplement le caractère hégémonique et définitif. Cette proposition permet de préciser un point : notre discussion a porté sur la possibilité pour l'éducateur de se référer au minimalisme moral pour conduire son action et non de considérer cette conception comme objet de transmission.

Les conceptions habituelles de l'éducation scolaire s'appuient sur une vision des tâches afférentes comme réduites à l'intervention directe des éducateurs sur les élèves. Cette façon de voir est extrêmement réductrice par rapport à l'ensemble des actions des professionnels pour favoriser la réussite de leurs élèves et en particulier celles qui leur font mobiliser des tiers pour atteindre cet objectif. A contrario, une conception de l'éducation scolaire conçue comme activité d'imputation induit la prise en compte des principes moraux des usagers et justifie un recours à des positions éthiques minimalistes ou maximalistes. La notion d'éthique minimale peut alors jouer à la fois un rôle descriptif et explicatif. Descriptif car elle permet de faire apparaitre les caractéristiques pluralistes de la pensée morale des éducateurs en montrant qu'elle peut comporter des dimensions plurielles. Explicatif car elle permet d'élaborer quelques composantes d'un modèle d'intelligibilité de cette pensée. Dans la partie suivante, nous préciserons cette idée en envisageant l'opportunité de considérer la pensée éthique des éducateurs comme relevant, dans certaines conditions et situations, d'un pluralisme d'exploration.

\section{Des éducateurs pluralistes?}

14 Cette possibilité des éducateurs d'être, suivant les situations, maximalistes ou minimalistes est-elle effective dans les pratiques quotidiennes? Pour le savoir, nous avons mené, dans le cadre d'une recherche doctorale (Lorius, 2015), des entretiens avec des professionnels au contact quotidien des élèves et de leurs familles (enseignants, chef 
d'établissement...). Nous avons cherché à savoir comment nos interlocuteurs se situaient face aux modalités d'implication des élèves et de leur famille dans le projet scolaire : la considèrent-ils comme un préalable à leur action ou comme un objectif possible de celleci ? En effet, les différentes façons de répondre à ces questions permettent de concevoir un éducateur qui serait plutôt minimaliste et dont on peut s'attendre à ce qu'il tolère, de la part des usagers, une contestation des dispositifs scolaires qui lui sont proposés ${ }^{7}$ au motif que cela relève de leur liberté. À l'inverse, un éducateur maximaliste jugera nécessaire et légitime de considérer les modalités de prise en charge définies par l'institution comme s'imposant aux usagers : ces deux éducateurs se différencient en ce qu'ils défendent ou rejettent la vision d'un état éducateur «permissif et parcimonieux dans l'usage de sa force » (Ogien, $2013: 268$ ).

En première analyse, on peut donc envisager deux manières d'aborder les questions d'imputation par les éducateurs scolaires. La première est de considérer que l'adhésion au projet scolaire, de la part des élèves ou de leur famille, est un préalable sur lequel il n'est ni possible, ni souhaitable d'agir. Cette position revient à appréhender l'obligation scolaire non seulement en terme de présence physique, mais également en termes d'investissement: ce qui serait obligatoire, c'est-à-dire imposé par la nation comme condition de l'appartenance à la communauté scolaire, ce serait une adhésion autant qu'une présence. Ce positionnement peut être considéré comme maximaliste, car se considérant légitimement en surplomb des usagers pour définir ce que serait, pour eux, le bien scolaire. Les éducateurs peuvent également s'en tenir à une conception plus modeste de l'obligation scolaire en se reconnaissant une responsabilité dans la transformation d'une obligation (de présence) en projet (d'apprentissage scolaire). Une conception de ce type induit naturellement l'acceptation de l'idée d'une adhésion des élèves et des familles à construire plutôt que présente d'emblée par le seul fait de la scolarisation ${ }^{8}$. Nous pouvons poser comme minimaliste cette seconde vision dans la mesure où elle considère que la décision des usagers d'adhérer ou non au projet scolaire leur appartient et doit être prise en compte par l'éducateur'.

16 Il est évident que les façons d'éduquer scolairement, c'est-à-dire d'imputer les ressorts de l'apprentissage, dépendront fortement du fait que l'on se réfère à l'une ou l'autre de ces manières de voir. Si le premier mode induira des positionnements visant à présenter aux usagers un bien scolaire à prendre ou à laisser et qui reposeront donc sur des imputations visant à faire appliquer (les consignes, le règlement, les modes de préparation des leçons, les comportements à promouvoir...), le second autorisera des méthodes conduisant à proposer, à échanger (autour des formes de prises en charge pédagogiques, de la place des parents au sein de celles-ci, sur l'opportunité des objectifs retenus...). Ces conceptions renvoient aux questions originelles de l'obligation scolaire qui opposaient la vision minimaliste de Nicolas de Condorcet ("Que nul ne puisse se plaindre d'avoir été écarté ») à une vision républicaine plus contraignante concevant un droit (à la fois universel et inconditionnel) à l'éducation : «Que nul ne puisse se plaindre d'avoir été obligé, puisque c'est pour son bien » (Monjo, 2013: 88). Reste que, effectivement, il n'est pas si simple d'admettre d'emblée l'opportunité et la possibilité de la transformation d'un droit inconditionnel en obligation et l'on peut se demander avec R. Monjo comment l'école peut être à la fois « émancipatrice et obligatoire ».

Nous avons argumenté en faveur de l'idée selon laquelle il n'était plus possible de définir le travail des éducateurs scolaires uniquement en fonction de la nature de leur intervention directe sur les élèves dont ils ont la charge. Des pans entiers de leur action 
seraient oubliés si l'on procédait de la sorte. Aujourd'hui c'est une forme de polyvalence qui caractérise le travail d'éducation scolaire. Il s'agit en effet d'éduquer dans une relation directe aux élèves mais également de coopérer avec les parents, avec des intervenants extérieurs... De fait, la responsabilité éducative devient partagée. Ce partage de responsabilité est «coloré" par l'obligation légale qui induit pour les élèves une présence mais pas nécessairement une activité. Les entretiens que nous avons menés pour comprendre comment, dans ce contexte, les deux options maximalistes et minimalistes impactent les pratiques, ont permis d'identifier plusieurs types de positionnements se situant entre des positions clairement maximalistes (sous-tendues par l'idée que les objectifs scolaires sont par définition légitimes), ou clairement minimalistes (sous-tendues par l'idée que les décisions des usagers sont premières et peuvent remettre légitimement en cause l'opportunité de certaines prises en charge scolaires). Nous avons également constaté que, au regard de ces différents indicateurs, les repères peuvent varier pour une même personne interrogée.

Pour établir ce résultat, nous avons par exemple proposé à nos interlocuteurs de réfléchir à la situation suivante :

«Comment réagiriez-vous face à un élève dont la description faite par le professeur principale au moment du conseil de classe serait : $\mathrm{X}$ a des résultats très faibles dans toutes les matières (la moyenne n'est franchie qu'en éducation physique). Les appréciations montrent à la fois des lacunes et un manque de travail. Ce dernier n'est ni assez soutenu, ni assez efficace. L'attitude passive pendant les cours renforce le problème. Quelques résultats (par exemple le dernier devoir de géographie) indiquent qu'une autre situation est possible $»^{10}$.

19 Face à cette proposition, nous avons constaté qu'à certains moments de l'entretien, une personne peut par exemple recourir à des positionnements maximalistes comme l'appel à une plus grande implication de ses parents. À d'autres moments, le même interlocuteur peut faire preuve d'une vision minimaliste où domine un principe de non-nuisance et l'amenant à considérer que, peut-être, face à un élève de ce type, «l'exhortation » ou la contrainte pédagogique ne seront pas efficaces ou justifiées. Ceci peut se traduire par des propos indiquant qu'il faut donner temporairement la priorité à la signification que l'élève ou les parents accordent à la scolarité et qui peut se manifester par un faible investissement. Les éducateurs peuvent donc recourir à des positionnements variés pour prendre en compte le rapport au projet scolaire des élèves et de leurs parents.

Ce pluralisme prend acte du fait que les réponses pédagogiques ne préexistent pas aux problèmes rencontrées dans la pratique. Les éducateurs font alors preuve de ce que l'on pourrait appeler un pluralisme d'exploration, qui permet d'envisager, sur le plan éthique, la situation sous des angles divers. Nos observations tendent donc à montrer que les éducateurs recourent à une valeur faible donnée aux principes et valeurs. En effet, le pluralisme qu'ils utilisent révèle que les principes moraux sur lesquels ils s'appuient varient: les obligations le deviennent si elles sont reconnues comme adaptées à la situation. Ce résultat est important et il est utile de s'attarder sur ce point ne serait-ce que pour vérifier que cette possibilité est envisageable sur le plan théorique.

Il est en effet possible qu'une conception forte de l'obligation morale ne soit pas toujours adaptée à l'action scolaire et un exemple nous permettra d'illustrer cette possibilité. Il n'est pas rare que, en début d'année scolaire ou après une période où les difficultés se sont accumulées dans un établissement, une équipe pédagogique considère urgent de rappeler le caractère imprescriptible, "non négociable», des éléments constitutifs du règlement intérieur. Cette volonté se traduit en général par le fait de déplorer le laxisme 
de tel ou tel (parents, direction, enseignant...) et par le caractère en général vain de cette approche. En effet, chaque journée «normale» dans un établissement scolaire « normal », apporte à la fois la preuve de la nécessité de règles communes permettant de créer une sécurité pour chacun, et d'arbitrages en situation : peut-on toujours reprocher à un élève de s'être défendu, y compris violement, face à une agression caractérisée ? Peut-on toujours reprocher à un élève d'avoir eu une attitude déplacée face à l'injustice criante d'un adulte? Ces "accrocs» ne remettent bien évidemment pas en cause l'opportunité d'obligations, mais celles-ci peuvent être, à certains moments, supplantées par des raisons liées aux situations. Une des manières d'expliquer pourquoi un positionnement du type « halte au laxisme » tel que présenté dans l'exemple précédent est inadapté, est de considérer que la mise en balance d'obligations relève de ce que nous appellerons la délibération-dilemme.

Or, délibérer en recourant à des dilemmes, c'est prendre le risque de refuser de concevoir que les mobiles éthiques peuvent être d'une autre nature que des obligations catégoriques. B. Williams (1990) a identifié la délibération-dilemme comme relevant non pas de la morale ou de l'éthique mais de la moralité, laquelle présente à ces yeux un grave défaut: cette dernière considère que les obligations morales ne peuvent être conflictuelles. Cela revient à dire que s'applique à la pensée morale une règle d'agrégation voulant que «si je suis obligé de faire $X$ et si je suis obligé de faire $Y$, je suis obligé de faire $\mathrm{X}$ et $\mathrm{Y}$ ». Pourtant, dans le sens ordinaire accordé à la notion d'obligation, qui n'est pas contrôlé par ces exigences particulières qui seraient spécifiques à la morale, il est évident que les obligations peuvent entrer en conflit.

L'éthique scolaire consiste à définir un préférable pour les élèves comme guide de l'action. Le recours à la pensée de B. Williams permet de comprendre que la détermination de ce préférable ne peut se faire à partir d'un panel d'obligations ou de principes qui permettraient d'en définir la nature quelle que soit la situation. Le principe, la valeur, sont donc des moyens et non des buts, et c'est pourquoi, dans le champ éducatif, les principes généraux ne valent que dans la mesure où ils sont support à une définition personnelle, en situation, de ce qui est bien ou mieux. Nous en tirons la conclusion que le repérage de positionnements minimalistes chez les éducateurs doit nous faire réfléchir sur le soi-disant risque qu'il y aurait à ne pas définir à priori les « bons » repères moraux éducatifs. Les éducateurs sont en effet et en pratique pluralistes au sens où l'entend D. Weinstock (2002), c'est-à-dire qu'ils peuvent concevoir une importante variété de croyances et de conceptions du bien scolaire. Ils sont donc pluralistes au sens où ils mobilisent des conceptions minimalistes ou maximalistes de leur activité : par la souplesse morale dont ils font preuve en situation, ils récusent l'idée d'une éducation fondée sur une liste finie et prédéterminée de principes moraux. $\mathrm{Ce}$ choix leur est nécessaire tout simplement pour pouvoir tenir leur rôle, en prenant appui sur les repères moraux en cours dans une société pluraliste. Bien sûr, cette position est difficile à tenir au quotidien car elle télescope une conception de l'éducation où les solutions éthiques sont censées préexister aux problèmes.

\section{Les textes réglementaires, un support pour s'exercer au pluralisme éducatif}

Dans cette dernière partie, nous illustrerons l'intérêt de ce pluralisme d'exploration en montrant comment il permet de transformer une contrainte (les textes réglementaires), 
en ressources pour l'action. Pour cela, nous essaierons d'envisager les conséquences concrètes que pourrait avoir, sur les pratiques professionnelles, le refus de lire les prescriptions institutionnelles uniquement à partir d'une liste de principes ou de valeurs devant, en tous lieux et en tous temps, guider les acteurs. Nous proposerons ainsi quelques arguments en faveur d'une évolution du regard porté sur les textes qui encadrent les pratiques éducatives, dans le but de favoriser des modalités de jugement et d'engagement pluralistes.

Pour R. Ogien, il est possible de différencier les énoncés normatifs et évaluatifs en considérant que les premiers ont un domaine d'application plus étroit que les seconds :

«Les énoncés normatifs portent généralement sur ce qu'il faut faire et s'appliquent à des actions humaines. Tout énoncé normatif devrait, en principe, faire référence à un certain genre d'action, préciser quels agents peuvent, doivent ou ne doivent pas l'accomplir et dans quelles circonstances. D'autre part, l'action ne doit être ni nécessaire ni impossible [...]. En revanche, les énoncés évaluatifs ne contiennent pas nécessairement de référence à une action et des précisions concernant des agents et des circonstances (et) peuvent s'appliquer à ce qui est nécessaire ou impossible » (Ogien, $2003:$ :108).

Nous pensons que cette distinction entre évaluatif et normatif peut être un critère de classification des textes qui encadrent la pratique des éducateurs scolaires. Ce critère permet en effet de différencier les énoncés qui rappellent un souhaitable, un préférable, de ceux qui précisent en quoi relève la responsabilité et les possibilités de chacun pour l'atteindre. Il est ainsi possible de positionner suivant ces critères les textes réglementaires qui seront plutôt évaluatifs ou normatifs suivant qu'ils tendent ou non vers le simple rappel de préférence ou d'objectifs ou qu'ils vont jusqu'à la proposition de mises en œuvre contextualisées à promouvoir ou proscrire. Ainsi, les dispositions contenues dans les textes réglementaires peuvent relever du normatif si elles sont suffisamment décrites et calibrées. Au contraire les rappels des grands principes éducatifs sont des énoncés évaluatifs.

Nous posons l'hypothèse selon laquelle ces textes reposent en général sur des énoncés évaluatifs même s'ils se veulent "normativistes" au sens où ils se posent comme normatifs alors même qu'ils ne donnent pas de précisions susceptibles d'organiser précisément les pratiques. Cette réalité est bien connue, y compris des institutions de contrôle de l'activité scolaire comme le montre l'extrait ci-dessous :

« Les textes normatifs sont à la fois détaillés et lacunaires. Ils ne portent pas en euxmêmes les conditions de leur traduction concrète et de leur adaptation aux différents environnements. Les séquences d'ajustement perpétuel, de réglage fin par essai et erreur échappent au regard du public. Cette tâche d'adaptation des normes nationales aux attentes locales, déléguée aux enseignants, reste invisible alors qu'elle est cruciale. L'accompagnement demeure trop souvent défaillant si bien que les enseignants se retrouvent souvent seuls, parfois rassemblés en groupes informels, pour répondre à la question centrale : que faire de telle ou telle mesure avec mes ressources et mes contraintes? ? (Gonthier-Maurin, $2012: 35$ )

La dernière circulaire de rentrée publiée au titre de la rentrée $2014^{11}$ est assez emblématique de ce point de vue. Elle est dans sa forme évaluative car il parait improbable que les destinataires de ce texte de 23 pages parviennent à mettre en œuvre l'ensemble des recommandations qui leurs sont adressées et présentées comme autant de priorités. Cette caractéristique évaluative est présente dans le contenu même de cette circulaire qui est porteuse de nombreux conflits d'obligations : entre des impératifs de mise en œuvre et le contexte général de réduction de moyen (comme lorsqu'il est fait 
mention au point I. 5 du rôle des centres d'information et d'orientation alors que ceux-ci sont progressivement démantelés) ou entre des fins contradictoires (comme la priorité accordée à certains moments à l'insertion professionnelles et à d'autres à la formation générale).

Nous avons montré plus haut que les questions d'éthique éducative ne pouvaient se réduire à l'arbitrage entre des principes. Nous référant à B. Williams, nous avons expliqué en quoi un recours modeste aux obligations était la condition d'une morale attentive aux situations, et ne se mettant pas elle-même dans des impasses logiques, et sans que ceci soit un obstacle à la pensée éthique. Cette option télescope celle retenue par le cadre réglementaire : contrairement à ce que ce dernier laisse sous-entendre, il est impossible de promouvoir la réussite scolaire de tous, au sens d'un accès de tous aux certifications, aux cursus scolaires et aux fonctions sociales les plus désirables. En effet, par définition, c'est un principe de concurrence qui s'applique à chacune de ces étapes. Ce que nous souhaitons souligner ici, c'est que les cadres dans lesquels s'insèrent les pratiques souffrent parfois d'un déficit de validité théorique, non parce qu'ils s'appuieraient sur des considérations erronées, mais parce qu'ils n'aident pas suffisamment à une mise en perspective des gestes professionnels. La volonté de proposer des fondements « inattaquables » aux recommandations fait prendre à celles-ci le risque d'un dogmatisme (qui prend parfois la forme d'un angélisme) pouvant générer une méfiance en raison d'un déficit de réalisme des justifications de l'action.

Nous émettons l'hypothèse selon laquelle on pourrait retrouver un grand nombre de ces impossibilités logiques dans la plupart des textes régissant l'action des professionnels. Nous pensons aussi bien aux textes de portée générale (code de l'éducation, circulaires d'organisation de la rentrée...) qu'à ceux dont la portée est plus locale comme par exemple les principes moraux ou valeurs mis en avant dans les premières pages des projets d'établissement. S'il ne nous parait pas utile de procéder à une analyse détaillée de ces textes pour établir leur caractère moraliste plus que moral, c'est que, par définition, ils ne formulent les questions éthiques que de manière substantielle en énonçant ce qu'il faut atteindre (par exemple l'égalité), ce qu'il faut promouvoir (par exemple le respect), mais jamais aux dépens de quoi ces idées doivent être promues (l'égalité contre la liberté, le respect contre l'obéissance ?).

Outre le faible souci de préciser leurs modalités concrètes de mise en œuvre et celui de considérer comme conciliables des priorités ou principes qui ne peuvent que s'exclure, la troisième caractéristique du cadre réglementaire est de se préoccuper de résultats globaux ne prenant que peu en compte les transformations opérées chez les élèves. On retrouve cette tonalité dans la plupart des textes de cadrage mais également, de façon marquée, dans les contrats d'objectifs signés entre les académies et les établissements scolaires Cette caractéristique évaluative est susceptible de générer un refus d'engagement des acteurs au motif de l'abandon du souci des élèves au profit d'indicateurs chiffrés. Par ailleurs, une quatrième et dernière caractéristique est bien connue des acteurs. Les textes se préoccupant par nature plus de la conformité des actes que de leurs effets, ils sont susceptibles de compliquer l'apparition de pratiques divergentes mais potentiellement efficaces.

Pour au moins ces quatre raisons (de centration sur les principes et de nonhiérarchisation de ceux-ci, de centration sur les résultats globaux et sur la conformité), nous pouvons poser l'hypothèse que les textes réglementaires sont moins normatifs qu'évaluatifs. Pour qu'ils puissent malgré tout servir de support au développement de 
pratiques satisfaisantes, il conviendrait que les destinataires soient en mesure de résister, d'un côté, à la tendance de ces cadres à promouvoir l'agitation, le dogmatisme, et, d'un autre côté, à leur propension à générer l'incrédulité ou le conformisme.

L'idéal serait évidemment que ces textes comportent en eux-mêmes une meilleure définition des conditions de l'agir (en indiquant qui doit agir, dans quelles conditions, en oscillant entre quels " préférables »). Dans l'état actuel des choses et à court terme, il est sans doute plus réaliste de proposer aux éducateurs de porter un regard « en creux » sur les prescriptions qui organisent leur action, c'est à dire de compléter par eux-mêmes les éléments manquants pour pouvoir normer leur activité et la transformer en pratiques. Il s'agirait qu'ils décident par eux-mêmes du degré d'urgence à retenir pour la mise en œuvre, de repérer ce qui garantit ou infirme la justification de l'action à entreprendre, ce qui peut être tenté sans risque pour les élèves et si les demandes nécessitent une modification radicale des pratiques, une simple reprise ou même de surseoir à leur prise en compte. Pour reprendre l'exemple de la dernière circulaire de rentrée, devant ce texte, les éducateurs peuvent rester légitimement circonspects pour déterminer quelles priorités retenir avec les classes dont ils ont la charge, voire même se demander ce qu'ils convient de faire avec les points qu'ils pensent inapplicables voir nocifs. Ainsi, à la lecture de l'item intitulé «favoriser la persévérance scolaire et poursuivre la lutte contre le décrochage ", peuvent-ils effectivement se sentir peu concernés s'ils ont la charge d'élèves que le manque de persévérance caractérise beaucoup moins que la force des déterminismes de toutes sortes qui s'appliquent à leurs trajectoires scolaires. Ils peuvent alors, en se référant à $F$. Dubet (2008), considérer qu'il est judicieux de laisser à ces élèves la possibilité du désinvestissement scolaire comme dernière possibilité de sauvegarde de l'estime de soi face à une institution qui n'est pas organisée pour les prendre véritablement en compte. C'est à ce légitime «jeu » avec les injonctions réglementaire que nous invitons les éducateurs. Celui-ci se justifie en effet par sa concordance avec le pluralisme pratique dont ils font preuve selon nous au quotidien, et qui les fait, à juste titre, alterner, suivant les situations, entre des postures maximalistes d'adhésion au cadre scolaire, et minimalistes qui les amènent à parfois être plus prudents.

\section{Conclusion}

Les processus institutionnels qui ont pour fonction d'orienter l'action des professionnels de l'éducation scolaire prétendent se référer à des objectifs assignés à l'école qui seraient à la fois partagés, opératoires et compatibles entre eux. Pourtant, si l'on y regarde de plus près, ces objectifs constituent des directions de travail parfois opposées, délicates à mettre en œuvre et porteuses de conceptions de l'éducation scolaire qui pourraient être légitimement interrogées. C'est peut-être pour cela qu'il n'existe pas à proprement parler de code formalisé de déontologie pour les fonctionnaires d'état exerçant au sein du service public d'éducation. C'est en effet dans un vaste corpus de textes (la constitution, les lois, décrets et arrêtés, les codes divers, la jurisprudence) que sont disséminés les éléments fixant les normes à respecter par les ces acteurs.

En tout état de cause, raisonner en termes d'obligations qui se télescopent impose de dire quelles sont celles que l'on ne retient pas dans une situation donnée. Annoncer comme objectif la réussite de tous, tout en mettant en place des processus de sélection scolaire où les déterminismes sociaux et familiaux jouent un rôle important, revient, en pratique, à brandir ces valeurs mais pas à les promouvoir. Face à ce risque d'inconséquence éthique, 
les éducateurs font preuve d'une forte adaptabilité morale en situation, laquelle leur permet de définir un préférable pour chacune d'elles.

Ce pluralisme en acte peut être à notre avis conforté par la manière d'appréhender les textes réglementaires en enrichissant leur valeur normative. C'est par une étude au cas par cas des possibilités normatives du cadre réglementaire, c'est-à-dire en définissant par eux-mêmes le degré d'infusion des recommandations dans les pratiques qu'il convient d'opérer, que les éducateurs pourront à la fois justifier et renforcer leur capacité à éduquer dans un monde pluraliste.

\section{BIBLIOGRAPHIE}

CHAVEL, S. (2011) : «L'imagination morale dans la philosophie contemporaine de langue anglaise », Revue philosophique de la France et de l'étranger, 4 (136), p. 543-562.

DUBET, F. (2008) : Faits d'école, Paris, Éd. de l'École des hautes études en sciences sociales. DURAND, G. \& FABRE, M. (dir.) (2014) : Recherches en éducation, 6, mars (en ligne : http:// www.recherches-en-education.net/spip.php?article167, consulté le 15/10/14).

GO, H. L. (2012) : « La normativité dans l'éducation », Les Sciences de l'éducation pour l'ère nouvelle, 1

(2), p. 77-94.

GONTHIER-MAURIN, B. (2012) : Rapport d'information du Sénat, 601, session ordinaire de 2011-2012 (en ligne : http://www.senat.fr/rap/r11-601/r11-6011.pdf, consulté le 01/12/14).

KERLAN, A. (2003) : Philosophie pour l'éducation. Paris, ESF Éd.

LORIUS, V. (2015) : Le courage d'éduquer, imagination morale et activité des éducateurs en contexte scolaire, Nancy, PUN-Éditions universitaires de Lorraine.

marzano, M. (2009) : Je consens donc je suis, Paris, Presses universitaires de France.

MONJo, R. (2013) : « L'école peut-elle être, à la fois, libératrice et obligatoire ? », Le Télémaque, 1

(43), p. 87-102.

ogIEN, R. (2003) : Le rasoir de Kant, Paris, Éd. de l'Éclat.

- (2004) : La panique morale, Paris, Grasset.

- (2007a) : La liberté d'offenser. Le sexe, l'art et la morale, Paris, Éd. La Musardine.

- (2007b) : L'éthique aujourd'hui, maximalistes et minimalistes, Paris, Gallimard.

- (2013) : L'État nous rend-il meilleurs?, Paris, Gallimard.

OGIEN, R. \& TAPPOLET, C. (2008) : Les concepts de l'éthique, Paris, Hermann.

PRAIRAT, E. (2012a) : «Considérations sur l'idée de norme », Les Sciences de l'éducation pour l'ère nouvelle, 1 (2), p. 33-50.

- (2012b) : « La norme, l'école et... l'acte d'enseigner ", Les Sciences de l'éducation pour l'ère nouvelle, $1(2)$, p. 7-16.

WEINSTOCK, D. (2002) : « Le pluralisme axiologique en philosophie politique contemporraine », in : L. Sosoe, E. Lomomba, C. Des lauriers \& J. Pelabay, Diversité humaine : démocratie, multiculturalisme 
et citoyenneté, Laval, Presses de l'Université Laval.

- (2006) : Profession éthicien, Montréal, Presses de l'Université de Montréal.

WiLliams, B. (1990) : L'éthique et les limites de la philosophie, trad. de l'anglais par M.-A. Lescourret, Paris, Gallimard.

\section{NOTES}

1. Dans cet article, nous concevrons la norme comme un arbitrage socialement partagé et présentant des caractéristiques de régularité et de possibilité. Nous verrons donc la norme comme facteur de sécurisation du monde mais également comme limitation des manières de lire le réel : la norme règle temporairement et socialement un conflit entre plusieurs manières de procéder. Comprendre une norme revient donc à chercher à comprendre la nature des difficultés ou des conflits qu'elle doit résoudre ou rendre moins dramatiques au moyen d'usages.

2. C'est par exemple la conception des familles qui décident de scolariser, comme la loi le leur permet, leurs enfants à domicile.

3. Circulaire $n^{\circ}$ 2010-106 du 15-7-2010, bulletin officiel de l'Éducation nationale $n^{\circ} 29$ du 22 juillet 2010.

4. Voir par exemple la note d'analyse Quelle organisation pour le soutien scolaire?, centre d'analyse stratégique, janvier 2013, $n^{\circ} 315$, p. 2.

5. Pour un point sur cette question, voir G. Durand et M. Fabre (2014).

6. Nous pensons en particulier à toute les « éducations à... » : éducation à la sécurité, éducation à la santé... et aux mobiles fréquemment avancés pour justifier les dispositifs (développer le goût de l'effort...).

7. Nous ne parlons ici que des dispositifs et non du respect de l'obligation scolaire, dont il serait intéressant de débattre, mais qui ne fait pas partie du champ de cette discussion.

8. Il est intéressant de noter que le cadre réglementaire régissant le principe d'obligation d'instruction oscille entre les registres normatif (au sens d'une action pour imposer la norme scolaire comme arbitrage social préférable concernant l'instruction des enfants) et pénal. Le premier se retrouve dans les dispositifs d'accompagnement des familles et le second dans les sanctions prévues en cas de manquements (rappels à la loi, signalements au procureur de la République...).

9. Dans la limite, comme nous l'avons indiqué plus haut, d'un constat de tort flagrant causé à l'élève.

10. Sur demande de l'interviewé, les informations complémentaires suivantes pouvaient être apportées : il s'agit d'un garçon en grande difficulté sociale ; le professeur principal a rencontré les parents qui ne sont pas surpris. Ils savent depuis longtemps que leur enfant ne fera pas de longues études ; cet élève a une passion : il est pompier volontaire et dispose déjà du premier niveau de certification.

11. Circulaire $n^{\circ}$ 2014-068 du 20-5-2014 publiée au Bulletin officiel $n^{\circ} 21$ du 22 mai 2014. 


\section{RÉSUMÉS}

L'objectif de cet article est de proposer quelques arguments en faveur de la thèse selon laquelle le pluralisme moral, non seulement n'est pas un obstacle à l'activité des éducateurs scolaires, mais constitue probablement l'une des conditions de son déploiement. Pour étayer cette proposition, nous explorerons l'intérêt de deux points d'appui théoriques pour comprendre l'activité éthique des éducateurs en contexte scolaire. Le premier concept que mobiliserons sera celui d'éthique minimale, dans la version qu'en propose R. Ogien. Il nous servira à définir ce que nous pouvons entendre par pluralisme dans le domaine de l'éducation scolaire. Ensuite, nous envisagerons les conséquences d'un recours à la distinction entre «morale " et "moralité » proposée par B. Williams. De cette opposition, nous retiendrons la possibilité que la délibération-dilemme qui voudrait que les éducateurs fassent des choix entre des obligations dont la valeur serait préexistante aux situations ne soit pas un mode de pensée exclusif, mais qu'il faut sans doute lui associer ce que nous appellerons un pluralisme d'exploration. Dans la dernière partie de notre texte, nous proposerons un exemple où ce pluralisme d'exploration peut être d'une grande utilité pour la prise en compte des textes réglementaires.

The purpose of this article is to offer some arguments in favor of the thesis that moral pluralism, not only does not hinder the activity of school teachers, but probably one of the conditions of its deployment. In support of this proposal, we explore the interest of two points of theoretical support for understanding business ethics educators in schools. The first concept is that we will mobilize the minimum ethics in the version that offers R. Ogien. It will help us to define what we mean by pluralism in the field of school education. Secondly, we will consider the consequences of an appeal to the distinction between "moral" and "morality" proposed by B. Williams. This opposition, we will retain the possibility that deliberation dilemma-who wants that educators make choices between bonds whose value would be to pre-existing conditions is not an exclusive mode of thought, but it should probably associate him what we call an exploration in the last part of our text pluralism, we propose an example of pluralism exploration can be very useful: the consideration of statutory instruments.

\section{INDEX}

Mots-clés : pluralisme, éducation scolaire, minimalisme moral, normes scolaires

Keywords : pluralism, school education, moral minimalism, academic standards

\section{AUTEUR}

\section{VINCENT LORIUS}

GRÉÉ, Universités de Bourgogne, Université Lyon 2 